\title{
A novel MC4R deletion coexisting with FTO and MC1R gene variants, causes severe early onset obesity
}

\author{
Vassos Neocleous, ${ }^{1 *}$ Christos Shammas, ${ }^{1 *}$ Marie M. Phelan, ${ }^{2}$ Pavlos Fanis, ${ }^{1}$ \\ Maria Pantelidou, ${ }^{3}$ Nicos Skordis, ${ }^{1,4,5}$ Christos Mantzoros, ${ }^{6}$ Leonidas A. Phylactou, ${ }^{1}$ \\ Meropi Toumba ${ }^{7,1}$
}

${ }^{1}$ Department of Molecular Genetics, Function \& Therapy, The Cyprus Institute of Neurology \& Genetics, Nicosia, Cyprus;
${ }^{2}$ Department of Biochemistry NMR Centre for Structural Biology, University of Liverpool, Institute of Integrative Biology,
Crown Street, Liverpool, UK; ${ }^{3}$ Department of Pharmacy and Department of Nursing, School of Health Sciences, Frederick
University, Nicosia, Cyprus; ${ }^{4}$ Division of Pediatric Endocrinology, Paedi Center for specialized Pediatrics, Nicosia, Cyprus; ${ }^{5}$ St
George's University of London Medical School at the University of Nicosia, Cyprus; ${ }^{6}$ Division of Endocrinology, Beth Israel
Deaconess Medical Center Boston, USA; ${ }^{7}$ Paediatrics \& Paediatric Endocrinology Clinic, IASIS Hospital, Paphos, Cyprus

*Equal contribution to this manuscript

\begin{abstract}
OBJECTIVE: Heterozygous mutations on the melanocortin-4-receptor gene (MC4R) are the most frequent cause of monogenic obesity. We describe a novel $M C 4 R$ deletion in a girl with severe early onset obesity, tall stature, pale skin and red hair. CASE REPORT: Clinical and hormonal parameters were evaluated in a girl born full-term by non-consanguineous parents. Her body mass index (BMI) at presentation ( 3 years) was $30 \mathrm{~kg} / \mathrm{m}^{2}$ (z-score: $\left.+4.5 S D S\right)$. By the age of 5.2 years, she exhibited extreme linear growth acceleration and developed hyperinsulinemia. METHODS: Direct sequencing of the MC4R, MC1R and for the known FTO single nucleotide polymorphism (SNP) rs9939609 was performed for the patient and her family. RESULTS: A novel heterozygous MC4R p.Met215del (c.643_645delATG) deletion was identified in the patient, her father and her brother, both of whom exhibited a milder phenotype. 3D structural dynamic simulation studies investigated the conformational changes induced by the p.Met215del. The patient and her mother were also found to be carriers of the obesity risk associated FTO rs9939609 SNP. Finally, the identification of the known p.Arg160Trp MC1R variant in the patient accounts for the red hair and pale skin phenotypic features. CONCLUSION: The p.Met215del causes global conformational and functional changes as it is localized at the alpha-helical transmembrane regions and the membrane spanning regions of the betabarrel. This novel mutation produces a severe overgrowth phenotype that is apparent as from infancy and is progressive in childhood. The additional negative effect of environmental and
\end{abstract}


unhealthy lifestyle habits as well as a possible co-interaction of FTO rs9939609 SNP may worsen the phenotype.

Key words: FTO gene, Melanocortin-4-receptor gene, Novel deletion, Obesity, Red hair

\section{INTRODUCTION}

Monogenic obesity accounts for $2-4 \%$ of obesity cases in children. ${ }^{1}$ Mutations on the melanocortin 4 receptor $(M C 4 R)$ are the most frequent monogenetic cause of human obesity. ${ }^{2,3}$ MC4R plays a critical role in body weight regulation through the leptin-melanocortin axis. It is a 332 amino acid protein encoded by a single exon gene localized on chromosome 18q22.32 (OMIM \#155541). It is expressed on the hypothalamic nuclei where it integrates a satiety signal provided by the alpha-melanocyte stimulating hormone (a-MSH) and an orexigenic signal provided by the Agouti related peptide (AGRP). ${ }^{4}$ Targeted deletion of the $M C 4 R$ results in severe obesity in homozygous mice, while heterozygous female animals have an intermediate body weight. ${ }^{5}$ Homozygous and heterozygous mutations on the $M C 4 R$ gene in humans may lead to hyperphagic and severe early onset obesity phenotypes. ${ }^{6}$

Genome-wide association studies (GWAS) have detected a strong association between single nucleotide polymorphisms (SNPs) within or near the MC4R gene as well as within the fat mass and obesity-associated gene $(F T O)$, and obesity various phenotypes. ${ }^{7,8}$ The FTO gene, located on chromosome 16q12.2 (OMIM \#610966), is another key gene that appears to be responsible for variations in body weight and composition. The FTO rs9939609 SNP have been highly associated with increased body mass; it has two alleles, $\mathrm{T}$ and $\mathrm{A}$, the latter being a variant associated with risk of obesity. AA homozygotic individuals are on average $3 \mathrm{~kg}$ heavier, with an increased BMI of about $0.8 \mathrm{~kg} / \mathrm{m}^{2}$, than those without the risk allele. ${ }^{9,10}$

The melanocortin 1 receptor $(M C 1 R)$ gene, located on chromosome 16q24.3 (OMIM \#155555), is expressed on melanocytes stimulating the synthesis of eumelanin after binding with a-MSH. The human $M C 1 R$ gene is highly polymorphic and certain allelic variants of the gene are associated with pale skin, red hair and skin cancer depending on the responses of human melanocytes to a-MSH and ultraviolet (UV) radiation. ${ }^{11-13}$

We report the phenotype/genotype of an obese girl screened for $M C 4 R$ and $M C 1 R$ gene mutations. The patient and her family were also participants in an FTO gene polymorphisms screening study.

\section{CASE REPORT AND METHODS}

\section{Patient}

A 3-year old girl presented with severe early onset obesity. She is the second child of non-consanguineous parents. The mother's pregnancy was uncomplicated followed by a cesarean section because of her high myopia. The patient was born full-term and was appropriate for gestational age in size (birth weight was $3.15 \mathrm{~kg}$; birth height was $50 \mathrm{~cm}$ and head circumference was $34 \mathrm{~cm}$ ) with no postnatal complications. She was breastfed during the first 6 months of life and was introduced to solid food at the age of 5 months. Her six-year older brother is also obese. He was presented at the age of 13.5 years with a BMI +2.1 SDS (z-score) and acanthosis nigricans. Retrospective auxological measurements for the brother showed a gradual increase in his BMI between the ages of 3.5-5 years from +1.8 SDS to +2.9 SDS (z-score) and then a gradual decrease until the age of presentation. However, he never exhibited extreme linear growth acceleration or signs of early sexual maturation. The father's BMI is approximately $33 \mathrm{~kg} / \mathrm{m}^{2}$, while the mother is overweight with a BMI $27 \mathrm{~kg} / \mathrm{m}^{2}$. The family has never followed a healthy lifestyle. The history of obesity and type 2 diabetes was positive in the first degree relatives of both parents. Informed consent was taken from the parents.

\section{Auxologic and metabolic parameters - Methods}

The patient's growth and metabolic parameters were evaluated every 4-6 months in the paediatric 
endocrinology clinic (MT). Growth charts in the growth analyzer (GA) 3.5 (Application Ed Dutch Growth Foundation, PO Box 23068, $3001 \mathrm{~KB}$, Rotterdam, The Netherlands) were used. Growth charts US 2000 (for height, weight and BMI) and The Netherlands 1997 (for waist circumference) were used as no Cyprus population charts are available for GA. Bone Age (BA) was assessed according to the Greulich and Pyle atlas. Blood samples were collected after an overnight fast. Routine biochemistry was measured by using the usual enzymatic assays. Plasma insulin, thyroid function, prolactin, ACTH, cortisol and 25-hydroxyvitamin $\mathrm{D}_{3}$ were determined by using the RIA method according to the manufacturers' instructions. IGF1 was measured with IGF1-RIACT (Schering) and IGFBP3 by IGFBP3-IRMA (Biocode).

\section{DNA amplification and sequence analysis}

Genomic DNA was isolated from peripheral whole blood and the desired regions were amplified by polymerase chain reaction (PCR) in reaction volumes of $20 \mu \mathrm{l}$ using 100 ng genomic DNA. The $M C 4 R$ (ENSG00000166603) gene was analyzed according to a cascade strategy and the entire coding sequence of 999 bps of the gene was initially amplified by PCR at $53{ }^{\circ} \mathrm{C}$ annealing temperature using the $M C 4 R$ Forward 1: 5' CCC TGA CCC AGG AGG TTA AA 3' and the $M C 4 R$ Reverse 2: 5' ACG GAA GAG AAA GCT GTT GC 3' primers. For the complete sequencing analysis of the entire coding sequence of the $M C 4 R$ gene the internal primers $M C 4 R \_$Reverse 1: 5'GCAAGC TGC CCA GAT ACA AC3' and $M C 4 R$ Forward 2: 5'TTT CAA TTG CAG TGG ACA GG3' were used. For the detection of the FTO (ENSG00000140718) gene of the SNP rs9939609, the set of primers FTO for: 5' GCT ATG GTT CTA CAG TTC CAG TCA T 3' and FTO rev: 5' AGG TCA GGA ATA ACC AGC TTA AAG T 3' were used and the desired region was PCR amplified at $60{ }^{\circ} \mathrm{C}$ annealing temperature.

Finally, for the identification of variants of the $M C 1 R$ (ENSG00000258839), the set of primers MC1R for: 5' CCT GGC AGC ACC ATG AAC TA 3' and MC1R rev: 5' GTA AGG AAC TGC CCA GGG TG 3 ' were used to amplify the entire coding sequence of the gene. The internal primers MC1Rint for: 5' CTT CTA CGC ACT GCG CTA CC 3' and MC1Rint rev: 5' ACG TGG TCG TAG TAG GCG AT 3' were also used for the complete sequencing analysis on an $\mathrm{ABI}$ 3130XL apparatus (Applied Biosystems, Waltham, MT, USA).

\section{In silico analysis of the novel MC4R deletion}

The in silico software of the computational algorithms Mutation Taster was used to predict the pathogenicity of the novel deletion.

\section{MC4R structural analysis}

3D structural dynamic simulation studies were used to investigate the conformational changes induced by this novel amino acid deletion in the MC4R protein structure.

\section{RESULTS}

\section{Phenotype}

The patient was firstly referred to the paediatric endocrine clinic for extreme weight gain and hyperphagia (Figure 1). The patient's psychomotor devel-

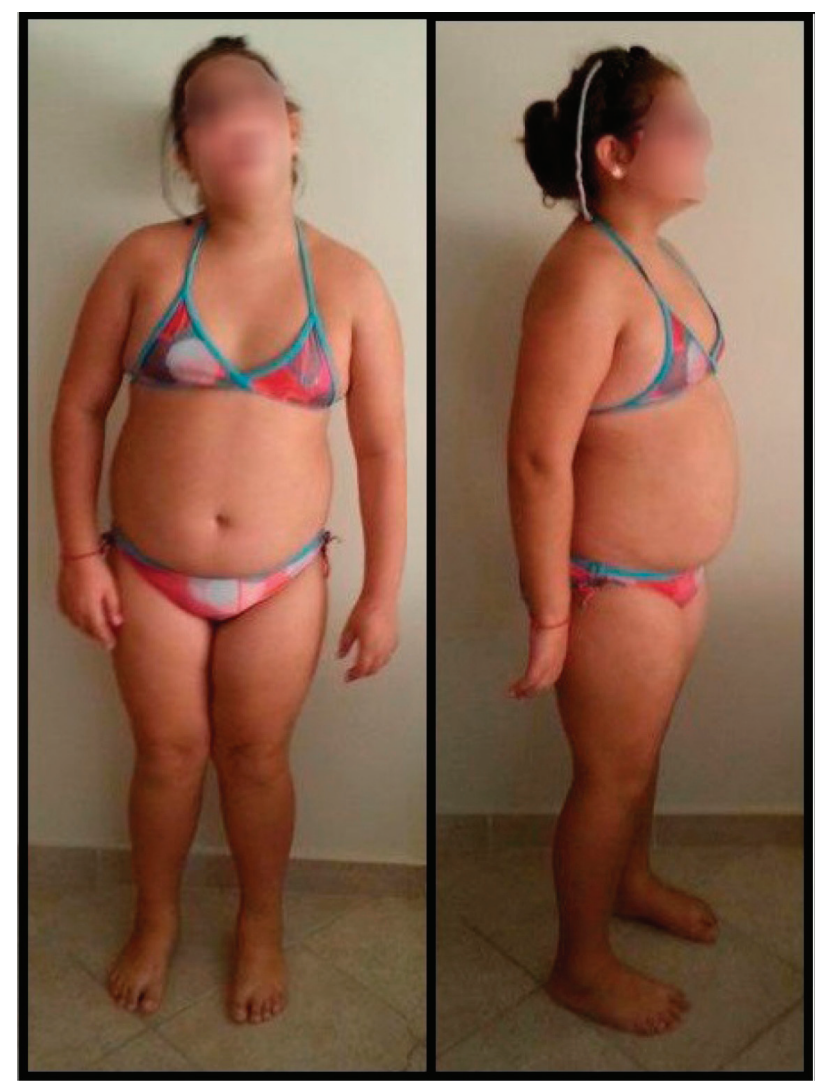

Figure 1. The photo shows the patient with severe early onset obesity, at the age of 6.5 years. 
opment was normal. She had no syndromic features and no muscle weakness but her red hair and pale skin were characteristic. She had no history of recurrent infections or sleep disorders. The patient's growth curves for height, weight, BMI and waist circumference are shown in Figure 2. Excessive weight gain started just after the age of 6 months. At the time of presentation (age 3 years), her BMI was $30 \mathrm{~kg} / \mathrm{m}^{2}$ (z-score: $+4.5 \mathrm{SDS})$, her waist circumference was 81 cm (z-score: +6.1SDS), while her BA was 2 years advanced compared to her chronological age (CA). At the age of 5.2 years, she exhibited extreme linear growth acceleration with growth velocity at $10.5 \mathrm{~cm} / \mathrm{y}$ and further BA advancement. Moderate acanthosis nigricans was noted in the cervical and axillary areas as well as a few abdominal striae. She had no signs of pubarche or thelarche. Additionally, she had moderate genu valgum deformity of the knees. Clinical and biochemical parameters are shown in Table 1. Liver and kidney function were normal as well as her thyroid function, morning cortisol, ACTH and prolactin levels. At the age of 5.2 years, her glucose/insulin ratio was 5.2 and her HOMA-IR was 2.4. The oral glucose tolerance test (OGTT) excluded diabetes mellitus and confirmed hyperinsulinemia. Her IGF1 and IGFBP3 were at higher than normal levels. Notably, she had low 25-hydroxyvitamin D3. The estimated daily calorie intake the patient received was about $2500 \mathrm{kcal}$ (in contrast to her age-matched normal daily needs of $1500-1700 \mathrm{kcal}$ ). She was advised to follow a special diet under the supervision of a clinical dietician. She was commenced on treatment with metformin 500mg per day due to hyperinsulinemia and also treated with vitamin D3 supplements. Six months after treatment she showed a slight improvement in her BMI. However, the family's attempt at creating for the child a healthy lifestyle by reducing calorie intake and increasing daily physical activity was not successful long-term.

\section{Genotype}

DNA sequencing in the affected girl presenting with severe early onset obesity identified the novel heterozygous p.Met215del (c.643_645delATG) mutation in the $M C 4 R$ gene. The same p.Met215del was identified in both her father and her brother (Figure 3 ). In addition to the novel p.Met $215 \mathrm{del}$ in the $M C 4 R$ gene, the patient inherited in heterozygosity the FTO SNP rs9939609 known to be linked with obesity risk and the p.Arg160Trp variant of the MC1R known to be associated with red hair and pale skin. Both FTO SNP rs9939609 and p.Arg160Trp of the MC1R were
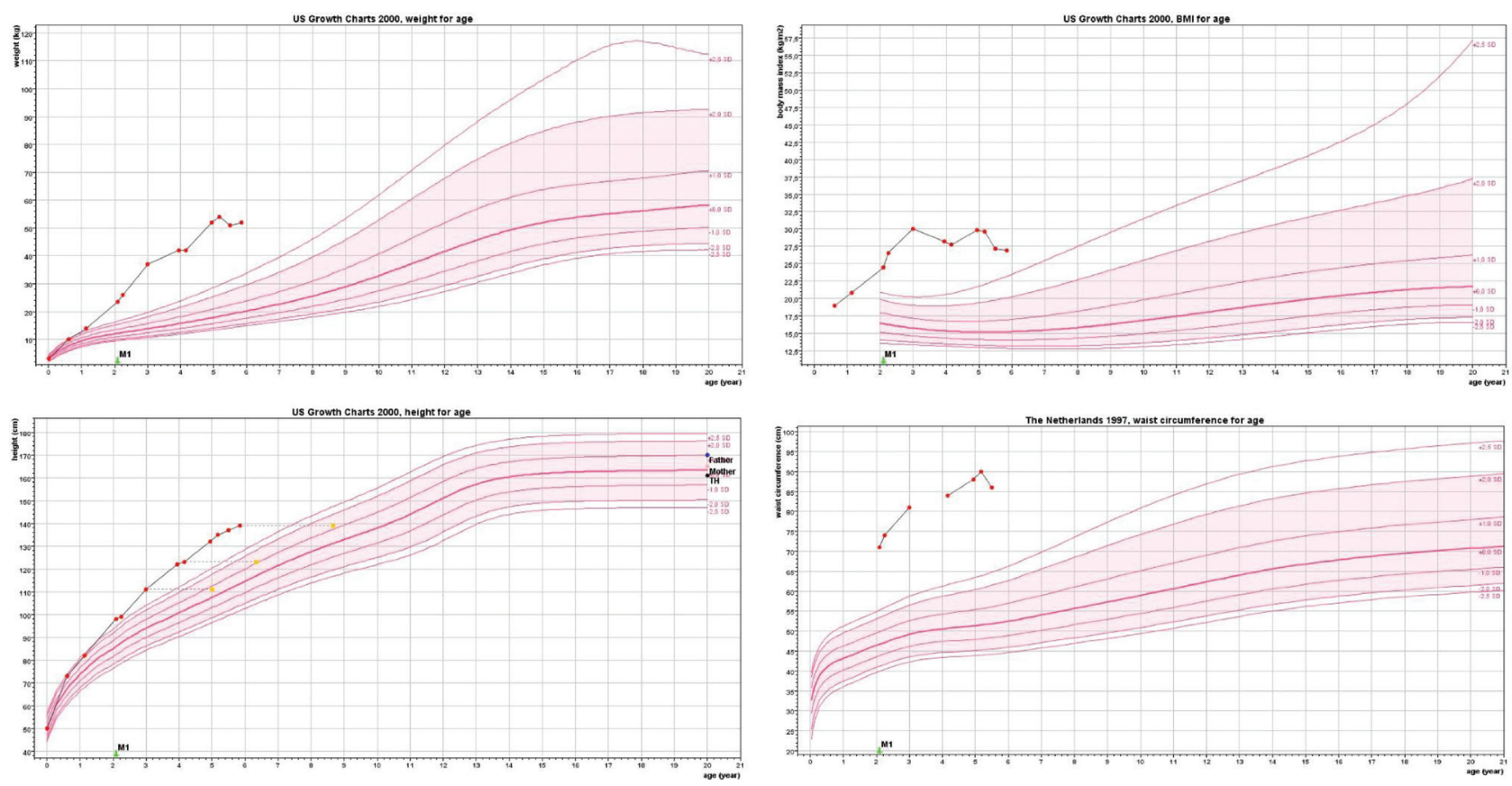

Figure 2. Growth charts of the patient with the novel $M C 4 R$ p.Met215del. 


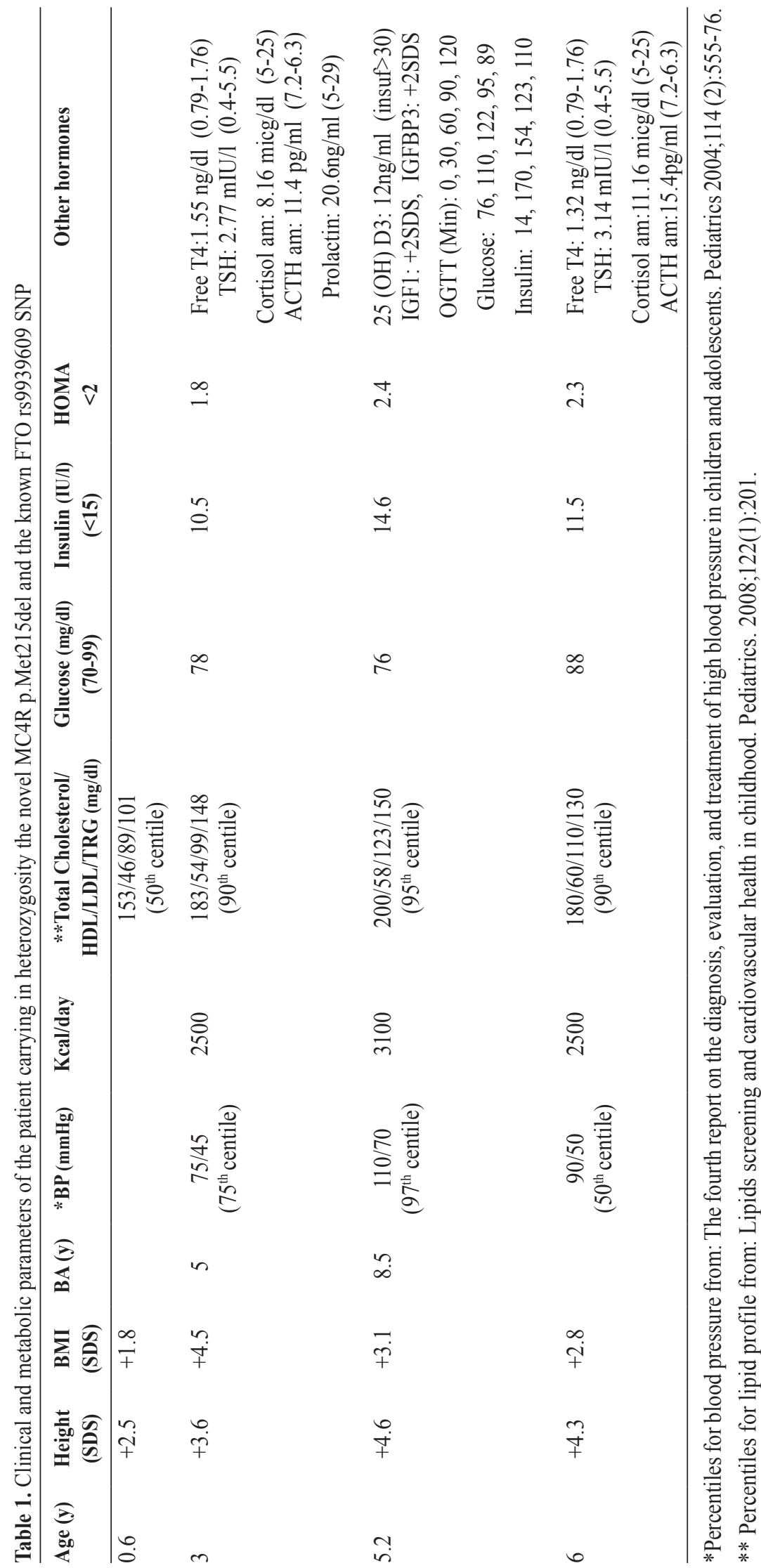

also observed in heterozygosity in the mother but not in the father or the brother of the proband. Another $M C 1 R$ variant, $p$. Val60Leu, was also found in our patient which is known to be associated with red hair but to a lesser extent than p.Arg160Trp.

\section{In silico analysis of the novel p.Met215del (c.643_645delATG)}

The novel heterozygous p.Met215del (c.643_645delATG) mutation is, according to the in silico computational algorithm Mutation Taster, predicted to interfere with protein function and is characterized as a disease-causing mutation.

\section{MC4R protein structure}

The $M C 4 R$ protein is a rhodopsin-like GPCR - cell surface receptor comprising a seven-transmembrane helical bundle. ${ }^{14}$ The helices, predicted to range between 21 and 27 amino acids in length, are connected by three extracellular and three intracellular loops with an extracellular $\mathrm{N}$ terminus and intracellular $\mathrm{C}$ terminus. The extracellular loops (loops 2, 4 and 6) are between 17 and 5 amino acids in length, suggestive of short protrusions from the membrane. The intracellular loops (loops 1, 3 and 6) are between 12 and 33 amino acids in length and thus are likely to protrude further from the membrane into the cytoplasm, characteristic of the rhodopsin-like family of protein $\mathrm{G}$ receptors (Figure 4).

The deletion mutation removes a methionine from the very end of helix 5, where the helix joins the loop. These amino acids are extremely important as they often exhibit amphipathic properties at 


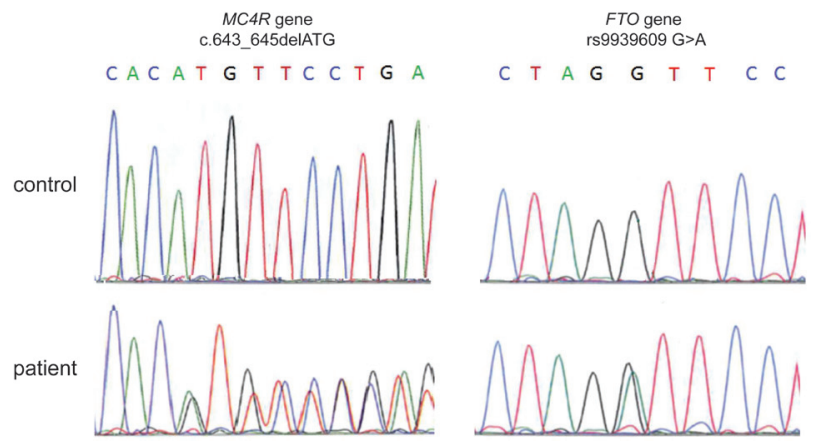

Figure 3. Electropherograms of the heterozygous patient carrying the novel $M C 4 R$ p.Met215del and the known FTO rs9939609 SNP

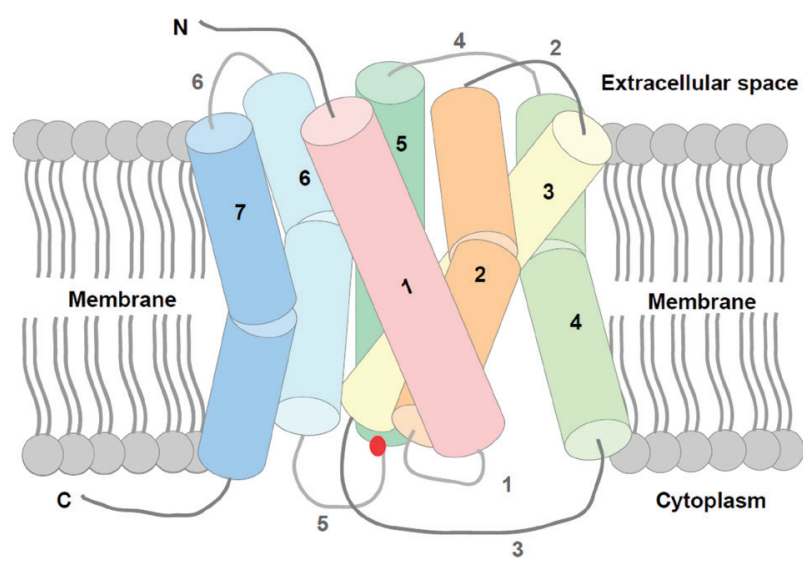

Figure 4. Schematic of $M C 4 R$ secondary structure based on schematic presentation of the rhodopsin class of GPCRs. Red spot indicates the site of deletion. Grey numbers indicate loop identities; numbers in black indicate helix identities.

the interface between hydrophobic membrane and aqueous cytoplasm. Furthermore, the site of deletion immediately precedes the longest cytoplasmic loop (loop 5).

\section{DISCUSSION}

Childhood obesity has become a dramatically increasing epidemic worldwide affecting more than $30 \%$ of children. ${ }^{15}$ Its rising prevalence and complex pathogenesis could be attributed to the interplay between dietary, environmental and genetic factors. ${ }^{16}$ This case report is a concrete example of the interaction between adipose regulatory genes and an unhealthy lifestyle resulting in morbid obesity.
Our patient's phenotype with hyperphagia, very early onset obesity, overgrowth, red hair and pale skin but normal ACTH and cortisol secretion led us to search for mutations in the main energy homeostasis gene $M C 4 R$ and in the pigmentation-related gene $M C 1 R$.

$M C 4 R$ is expressed predominantly in the hypothalamus and is involved in energy homeostasis together with $M C 3 R$. MC1R is expressed by cutaneous melanocytes, where it has a key role in determining skin and hair pigmentation. MC2R is the classical adrenocortical ACTH receptor and mediates the effects of ACTH on steroid secretion, while MC5R mainly participates in exocrine gland function. ${ }^{17}$ The latter two were not associated with our patient's phenotype and thus were not tested.

We detected a novel heterozygous mutation $M C 4 R$ p.Met215del (c.643_645delATG) deletion which was also found in the father and brother. Multiple different missense and nonsense mutations in $M C 4 R$, which function through different mechanisms, have been reported, largely in subjects with severe obesity commencing in childhood..$^{18}$ Although homozygotes are found to be heavier than heterozygotes, foodseeking behavior and body weight regulation depend on the amount of functional MC4R. ${ }^{6}$ The 3D structural dynamic simulation studies used to investigate the conformational changes induced by this novel MC4R amino acid deletion have shown distinct conformational changes in the protein structure. More specifically, the p.Met215del shortens transmembrane helix 5 and this may have a profound effect on its function. This mutation occurs on the cytoplasmic side of the channel at the point at which the helix joins the largest cytoplasmic loop, which could have a role in recruiting the transport molecule. These channels need specific amino acids to facilitate the transfer of ions through them; although M215 has not previously been associated with a specific feature, we might infer a specific role for this residue based on the clinical presentation. Similarly, a couple of other studies reported MC4R deletion mutations causing phenotypes comparable to our patient's. ${ }^{19,20}$

Our patient exhibited increased linear growth with very early BA advancement. Insulin growth factors (IGFs) were found to be at the upper limit of the normal range. Although studies showed no evidence 
of excessive growth hormone $(\mathrm{GH})$ secretion due to MC4R deficiency, GH pulsatility is maintained, suggesting a role for MC4R in controlling hypothalamic somatostatinergic tone. ${ }^{21}$ Hyperinsulinemia, which may appear before the onset of obesity in these patients, is an additional factor that contributes to accelerated linear growth. ${ }^{22}$ However, the brother, who was found to carry the same mutation, has never shown extreme linear growth, despite the fact that he also developed early hyperinsulinemia. Thus, the overgrowth phenotype may be attributed to more complicated mechanisms generated by various genotypic aberrations. Farooqi et al observed that the MC4R mutation-affected phenotype becomes less prominent with age. ${ }^{6}$ This is an unexplained phenomenon, which is also observed in the brother whose BMI gradually decreased with age.

On the other hand, the obesity phenotype exhibited by the index patient of the present study might be affected by additional factors. It should be noted that she has been following a very high calorie diet and has not exercised since early childhood. Additionally, she was found to be a carrier of FTO rs9939609 SNP. The association of this variant with BMI was confirmed in large population studies involving both adults and children. ${ }^{9}$ Studies in children suggested that the effect of the FTO genotype on BMI becomes evident only after the age of 7 years and that it is not directly associated with energy intake or physical activity. ${ }^{23}$ More recently, Hardy et al observed that the associations of FTO rs9939609 on body size varied with age, i.e. the effect increased during childhood and adolescence and diminished in adulthood. ${ }^{7}$ This finding has been confirmed in the reported patient and her family. The mother, who was also a FTO rs9939609 carrier, had a higher BMI in adolescence and, from obese, became overweight in adulthood despite continuing to follow unhealthy habits.

Finally, our patient was found to be heterozygous for the known p.Arg160Trp variant of the MC1R gene associated with fair skin and red hair, phenotypic features also apparent in our patient. ${ }^{12,24}$ In vitro experiments have demonstrated the missense p.Arg 160 Trp variant as a possible sensitizing factor of human melanocytes when exposed to UV radiation by decreasing eumelanin and increasing pheomelanin production. ${ }^{13}$ Although in some studies this variant was found to be common in melanoma patients and to increase the risk of melanoma more than twofold, ${ }^{25}$ in a recent Greek study it was not found to improve melanoma risk prediction when added to a clinical nongenetic model.${ }^{26}$ Another $M C 1 R$ variant, p.Val60Leu, which was observed in our patient, is a very frequent polymorphism among Caucasians and is also found to be associated with red hair color. ${ }^{27}$

This case report clearly illustrates the complexity and variability of the evolving obesity phenotype among members of the same family due to a novel $M C 4 R$ mutation and its interaction with other genetic traits and environmental modifiers. It will be of great interest to see whether the phenotype of our patient is weakening or worsening with age. As there is not a one to one correlation between phenotype and genotype, it is possible that other genes and variants potentially influence her phenotype.

\section{ACKNOWLEDGEMENTS}

This work was supported by the A.G. Leventis Foundation.

\section{CONFLICT OF INTEREST STATEMENT}

There are no conflicts of interest to declare.

\section{REFERENCES}

1. Sabin MA, Werther GA, Kiess W, 2011 Genetics of obesity and overgrowth syndromes. Best Pract Res Clin Endocrinol Metab 25: 207-220.

2. Vaisse C, Clement K, Guy-Grand B, Froguel P, 1998 A frameshift mutation in human MC4R is associated with a dominant form of obesity. Nat Genet 20: 113-114.

3. Tan K, Pogozheva ID, Yeo GS, et al, 2009 Functional characterization and structural modeling of obesity associated mutations in the melanocortin 4 receptor. Endocrinology 150: 114-125.

4. Cone RD, Lu D, Koppula S, et al, 1996 The melanocortin receptors: agonists, antagonists, and the hormonal control of pigmentation. Recent Prog Horm Res 51: 287-317; discussion 318.

5. Huszar D, Lynch CA, Fairchild-Huntress V, et al, 1997 Targeted disruption of the melanocortin-4 receptor results in obesity in mice. Cell 88: 131-141.

6. Farooqi IS, Keogh JM, Yeo GS, et al, 2003 Clinical spectrum of obesity and mutations in the melanocortin 4 receptor gene. N Engl J Med 348: 1085-1095.

7. Hardy R, Wills AK, Wong A, et al, 2010 Life course 
variations in the associations between FTO and MC4R gene variants and body size. Hum Mol Genet 19: 545552.

8. Loos RJ, Lindgren CM, Li S, et al, 2008 Common variants near MC4R are associated with fat mass, weight and risk of obesity. Nat Genet 40: 768-775.

9. Frayling TM, Timpson NJ, Weedon MN, et al, 2007 A common variant in the FTO gene is associated with body mass index and predisposes to childhood and adult obesity. Science 316: 889-894.

10. Dina C, Meyre D, Gallina S, et al, 2007 Variation in FTO contributes to childhood obesity and severe adult obesity. Nat Genet 39: 724-726.

11. Valverde P, Healy E, Sikkink S, et al, 1996 The Asp$84 \mathrm{Glu}$ variant of the melanocortin 1 receptor (MC1R) is associated with melanoma. Hum Mol Genet 5: 16631666.

12. Box NF, Wyeth JR, O'Gorman LE, Martin NG, Sturm RA, 1997 Characterization of melanocyte stimulating hormone receptor variant alleles in twins with red hair. Hum Mol Genet 6: 1891-1897.

13. Scott MC, Wakamatsu K, Ito S, et al, 2002 Human melanocortin 1 receptor variants, receptor function and melanocyte response to UV radiation. J Cell Sci 115: 2349-2355.

14. Ellis C, Smith A, 2004 Highlighting the pitfalls and possibilities of drug research. Nat Rev Drug Discov 3: 238-278.

15. Lobstein T, Jackson-Leach R, Moodie ML, et al, 2015 Child and adolescent obesity: part of a bigger picture. Lancet 385: 2510-2520.

16. Albuquerque D, Stice E, Rodriguez-Lopez R, Manco L, Nobrega C, 2015 Current review of genetics of human obesity: from molecular mechanisms to an evolutionary perspective. Mol Genet Genomics 290: 1191-1221.

17. Gantz I, Fong TM, 2003 The melanocortin system. Am J Physiol Endocrinol Metab 284: E468-474.

18. Yeo GS, Lank EJ, Farooqi IS, et al, 2003 Mutations in the human melanocortin-4 receptor gene associated with severe familial obesity disrupts receptor function through multiple molecular mechanisms. Hum Mol Genet 12: 561-574.

19. Donohoue PA, Tao YX, Collins M, et al, 2003 Deletion of codons 88-92 of the melanocortin-4 receptor gene: a novel deleterious mutation in an obese female. J Clin Endocrinol Metab 88: 5841-5845.

20. Turner L, Gregory A, Twells L, Gregory D, Stavropoulos DJ, 2015 Deletion of the MC4R gene in a 9-year-old obese boy. Child Obes 11: 219-223.

21. Martinelli CE, Keogh JM, Greenfield JR, et al, 2011 Obesity due to melanocortin 4 receptor (MC4R) deficiency is associated with increased linear growth and final height, fasting hyperinsulinemia, and incompletely suppressed growth hormone secretion. J Clin Endocrinol Metab 96: E181-188.

22. Fan W, Dinulescu DM, Butler AA, et al, 2000 The central melanocortin system can directly regulate serum insulin levels. Endocrinology 141: 3072-3079.

23. Hakanen M, Raitakari OT, Lehtimaki T, et al, 2009 FTO genotype is associated with body mass index after the age of seven years but not with energy intake or leisure-time physical activity. J Clin Endocrinol Metab 94: 1281-1287.

24. Smith R, Healy E, Siddiqui S, et al, 1998 Melanocortin 1 receptor variants in an Irish population. J Invest Dermatol 111: 119-122.

25. Palmer JS, Duffy DL, Box NF, et al, 2000 Melanocortin-1 receptor polymorphisms and risk of melanoma: is the association explained solely by pigmentation phenotype? Am J Hum Genet 66: 176-186.

26. Stefanaki I, Panagiotou OA, Kodela E, et al, 2013 Replication and predictive value of SNPs associated with melanoma and pigmentation traits in a Southern European case-control study. PLoS One 8: e55712.

27. Beaumont KA, Newton RA, Smit DJ, et al, 2005 Altered cell surface expression of human MC1R variant receptor alleles associated with red hair and skin cancer risk. Hum Mol Genet 14: 2145-2154. 